\title{
Association between Nicotinamide Phosphoribosyltransferase and de novo Lipogenesis in Nonalcoholic Fatty Liver Disease
}

\author{
Bahareh Amirkalali $^{a} \quad$ Masoud Reza Sohrabi ${ }^{a} \quad$ Ali Esrafily ${ }^{b}$ Mahmoud Jalali ${ }^{c}$ \\ Ali Gholami $^{d, e}$ Payam Hosseinzadeh ${ }^{a}$ Hossein Keyvani ${ }^{f}$ Farzad Shidfarg $^{9}$ \\ Farhad Zamani ${ }^{a}$ \\ ${ }^{a}$ Gastrointestinal and Liver Disease Research Center (GILDRC), Iran University of Medical Sciences, Tehran, \\ ${ }^{b}$ Department of Environmental Health Engineering, School of Public Health, Iran University of Medical Sciences, \\ Tehran, and ' Department of Nutrition and Biochemistry, School of Nutritional Sciences and Dietetics, Tehran \\ University of Medical Sciences, Tehran, ${ }^{\mathrm{d}}$ Department of Public Health, School of Public Health, Neyshabur University \\ of Medical Sciences, Neyshabur, ${ }^{e}$ Department of Epidemiology, School of Public Health, Iran University of Medical \\ Sciences, Tehran, ${ }^{\mathrm{f}}$ School of Medicine, Iran University of Medical Sciences, Tehran, and ${ }^{9}$ Department of Nutrition, \\ School of Public Health, Iran University of Medical Sciences, Tehran, Iran
}

\section{Significance of the Study}

- Higher serum nicotinamide phosphoribosyltransferase (NAMPT) in women was associated with a lower hepatic de novo lipogenesis (DNL) index, while in men it was associated with higher hepatic fat and had no association with the DNL index. This finding could help the interpretation of the serum NAMPT level for nonalcoholic fatty liver disease prognosis, which is probably sex dependent.

\section{Keywords}

Nicotinamide phosphoribosyltransferase $\cdot$ Nonalcoholic fatty liver · De novo lipogenesis

\begin{abstract}
Objective: This study explored the association between serum nicotinamide phosphoribosyltransferase (NAMPT) and hepatic de novo lipogenesis (DNL) in nonalcoholic fatty liver disease (NAFLD) and determined whether or not this association is sex dependent. Subjects and Methods: In this cross-sectional study, 62 consecutive patients ( 32 males, 30 females) with NAFLD were recruited. Serum NAMPT (by ELI$\mathrm{SA})$, palmitic acid, and the DNL index of erythrocyte membranes as markers of hepatic DNL (by gas chromatography)
\end{abstract}

\begin{tabular}{|c|c|}
\hline KARGER & $\begin{array}{l}\text { Karger } \\
\text { Open access }\end{array}$ \\
\hline $\begin{array}{l}\text { E-Mail karger@karger.com } \\
\text { www.karger.com/mpp }\end{array}$ & $\begin{array}{l}\text { This is an Open Access article licensed under the terms of the } \\
\text { Creative Commons Attribution-NonCommercial } 3.0 \text { Un- } \\
\text { ported license (CC BY-NC) (www.karger.com/OA-license), } \\
\text { applicable to the online version of the article only. Distribu- } \\
\text { tion permitted for non-commercial purposes only. }\end{array}$ \\
\hline
\end{tabular}

were analyzed. The controlled attenuation parameter (CAP) and body impedance analyzer were used to assess hepatic and body fat, respectively. Univariate and multiple linear regressions (to adjust for confounders) were used to analyze the association of serum NAMPT with palmitic acid, DNL index, CAP, and body fat. Results: The respective values of serum NAMPT ( $2.44 \pm 1.03$ vs. $2.45 \pm 1.13 \mathrm{ng} / \mathrm{mL}, p=0.98)$, DNL index (3.11 [2.60-3.71] vs. 3.05 [2.40-3.59], $p=0.90)$, and palmitic acid (20.55\% [15.34-24.04] vs. 22.64\% [21.15-25.95], $p=0.07$ ) were not significantly different between men and women, but those of CAP (326 [300-340] vs. 300 [261.25$329], p=0.002)$ and body fat ( $37.71 \pm 3.80$ vs. $26.60 \pm 5.70$, $p<0.001$ ) were significantly higher in women. In women, serum NAMPT had a significant negative association with the DNL index $(\beta=-0.56, p=0.01)$. The DNL index also had 
a significant negative association with body fat $(\beta=-0.46, p$ $=0.02$ ). In men, the only significant association was the positive association between serum NAMPT and CAP ( $\beta=0.35, p$ $=0.035)$. Conclusion: Higher serum NAMPT in women was associated with a lower hepatic DNL index, while in men it was associated with higher hepatic fat and had no association with the DNL index. Therefore, the serum NAMPT level interpretation for NAFLD prognosis is probably sex dependent.

(c) 2017 S. Karger AG, Basel

\section{Introduction}

Nonalcoholic fatty liver disease (NAFLD) is a common liver disease worldwide [1]. The increase in unhealthy lifestyles has contributed to the increase in the prevalence of this disease [2]. The disease may progress from simple steatosis to nonalcoholic steatohepatitis (NASH), which is hepatic steatosis accompanied by inflammation or liver cell injury with or without fibrosis [3]. NASH can lead to fibrosis and cirrhosis and ultimately to hepatocellular carcinoma [4].

De novo lipogenesis (DNL) is known to be high in individuals with NAFLD and contributes to about $26 \%$ of liver lipids [5]. In this pathway, fatty acid synthase (FAS) catalyzes the formation of palmitic acid from malonyl$\mathrm{CoA}$ and acetyl-CoA [6]. Acetyl-coenzyme A synthetase (ACS) is another enzyme in this pathway which contributes to the formation of acetyl coenzyme A (palmitic acid precursor) [7].

Studies have shown that both of these enzymes are upregulated by nicotinamide phosphoribosyltransferase (NAMPT), which was first discovered in 1994 as a molecule with cytokine functions [8]. Later it was reported that this molecule has an important enzymatic role in the production of nicotinamide adenine dinucleotide (NAD) and is a regulatory factor for NAD-consuming enzymes such as sirtuins (SIRT) in different metabolic pathways $[9,10]$. The NAMPT is important for the formation of acetyl coenzyme A by providing NAD for SIRT3 to activate ACS [7]; FAS is also upregulated in differentiated adipocytes when incubated with NAMPT [10] and NAMPT inhibition significantly reduces the level of de novo synthesized myristic and palmitic acid in prostate cancer cells $[10,11]$.

A few in vitro studies have shown a possible role for NAMPT in the metabolic pathway of DNL $[10,11]$, but there has not been any in vivo study on the role of serum NAMPT as a marker for DNL levels in patients with
NAFLD. Previous studies indicated that DNL has different effects on the progression of NAFLD depending its source (liver or adipose tissue). Hepatic DNL causes a fatty liver and insulin resistance while adipose tissue DNL is negatively correlated with these factors [12]. It is important to determine which source of DNL correlates more closely with serum NAMPT.

It was reported that the fatty acid profile of erythrocyte membranes is a reliable biomarker of derangements in the liver lipid metabolism [13]. The DNL index (palmitic to linoleic acid ratio) has been suggested for assessment of the rate of palmitic acid synthesis in humans [14]; an increase in DNL indicates an increased ratio of palmitate to linoleate (an essential fatty acid). Hence, the present study explored the association between serum NAMPT and markers of hepatic DNL (erythrocyte membrane DNL index and palmitic acid level) in patients with NAFLD. Also, because sex hormones affect the expression and performance of adipokines [15], this association was evaluated in both sexes.

\section{Subjects and Methods}

\section{Design and Sample}

In this cross-sectional study, patients with NAFLD referred to the Liver Disease Clinic at Firoozgar Hospital in Tehran, Iran (who met both inclusion and exclusion criteria) were recruited consecutively. The study was approved by the Ethics Committee of the Iran University of Medical Sciences (Tehran, Iran) and was carried out in accordance with the Helsinki declaration (2013). All participants gave their written informed consent.

The inclusion criteria were 18 years of age, a diagnosis of steatosis based on ultrasonographic findings and a controlled attenuation parameter (CAP) of above 180 (5\%) on a fibroscan [16], a stable body weight $( \pm 2 \%)$, and physical activity for at least 3 months. The exclusion criteria were a liver stiffness measurement above 10 in the fibroscan [17], diabetes (fasting blood sugar $\geq 126$ $\mathrm{mg} / \mathrm{dL}$ or use of blood glucose-lowering drugs), drug abuse, exposure to chemical pollutants, use of lipid-lowering drugs, use of steatogenic or hepatotoxic drugs (amiodarone, calcium channel blockers, perhexiline maleate, tamoxifen, chloroquine, methotrexate, corticosteroids, synthetic estrogens), use of drugs that affect weight (antidepressants, antipsychotics or hormone therapy), antioxidants and polyunsaturated fatty acid supplements in the 6 months prior to the study, an endocrine disease that affects weight (such as hyperprolactinemia, Cushing syndrome, thyroid disorders, congenital adrenal hyperplasia), kidney or heart disease, other acute or chronic liver diseases such as viral hepatitis or cirrhosis, or a history of alcohol intake ( $>20 \mathrm{~g} /$ day).

Subjects

The included subjects were 62 patients with NAFLD, of which 32 were men and 30 were women. The participants were between 18 and 67 years of age. 


\section{Diagnosis of Fatty Liver in Sonography}

Sonography was used to diagnose a fatty liver as an increase in hepatic echogenicity, using renal echogenicity as a reference, enlargement of the liver, and a lack of differentiation of the periportal and bile duct wall reinforcement because of advanced hyperechogenicity of the parenchyma [18].

\section{Fibroscan}

Liver stiffness ( $\mathrm{kPa}$ correlated to fibrosis) was assessed using FibroScan (Echosens, France), an ultrasound-based vibrationcontrolled transient elastography device. Ten measurements of stiffness $(\mathrm{kPa})$ were performed by pressing a probe between the ribs. The median of the 10 measurements was compared with the designated values from the fibroscan scoring card. Fibroscan also quantifies steatosis using CAP, a measurement of ultrasound attenuation correlated to the decrease in the amplitude of ultrasound waves as they spread through the liver. Because fat affects ultrasound broadcasts, an increase in steatosis will result in a higher CAP value. The final CAP value was the median of the individual measurements that ranged from 100 to 400 decibels per meter $(\mathrm{dB} / \mathrm{m})$ [17].

\section{Medical History, Dietary Alcohol Intake, and Body Fat Mass}

Each participant was given a medical history questionnaire to complete. As the macronutrient composition of diet affects DNL [19], the habitual dietary intake of the participants was assessed using the semiquantitative food frequency questionnaire consisting of 168 items that was validated on a sample of healthy population [20]. The physical activity level was assessed using the long form of the international physical activity questionnaire (IPAQ) [21]. Alcohol intake was estimated by recording the type of alcohol consumed, its volume, and the frequency of consumption. A fatty liver was considered nonalcoholic if the patient consumed less than $20 \mathrm{~g}$ of alcohol per day.

Body fat mass was measured using a body composition monitor (BF511; Omron, Kyoto, Japan). This device measures the percentage of body fat by bioelectrical impedance.

\section{Anthropometric Measurements}

Weight, height, waist, and hip circumferences were measured according to standard protocols [22]. Body mass index (BMI) was calculated as body weight $(\mathrm{kg})$ divided by the square of height $(\mathrm{m})$ as $\mathrm{kg} / \mathrm{m}^{2}$.

\section{Biochemical Measurements}

A 12-h fasting venous blood sample was drawn from each participant to measure the biochemical parameters. Fasting blood sugar was determined by enzymatic procedures (Pars Azmon, Tehran, Iran) and serum insulin (Diaplus, Canada) and NAMPT levels by enzyme-linked immunosorbent assay (ELISA; BioVendor, Czech Republic). Hepatitis B surface antigens, hepatitis B surface antibodies, hepatitis $B$ core antibodies, hepatitis $C$ virus antibodies, and antinuclear antibodies were evaluated using third-generation ELISA kits (Acon, San Diego, CA, USA). Insulin resistance was assessed using the HOMA-IR index as follows: HOMA-IR = $($ glucose $[\mathrm{mg} / \mathrm{dL}] \times$ insulin $[\mathrm{mU} / \mathrm{L}]) / 405$.

Fatty Acid Measurement in Erythrocyte Membrane

Venous blood samples were centrifuged at 3,000 $\mathrm{g}$ for $10 \mathrm{~min}$ at $4{ }^{\circ} \mathrm{C}$ immediately after withdrawal and the erythrocytes were separated. An equal volume of physiological saline (sodium chloride $0.9 \%$ ) was added to the erythrocytes, shaken, and then centrifuged at $3,000 \mathrm{~g}$ for $10 \mathrm{~min}$ at $4^{\circ} \mathrm{C}$. The erythrocytes were washed using this procedure 3 times.

The washed erythrocytes were aliquoted and stored at $-80^{\circ} \mathrm{C}$. A sample $200 \mu$ l of washed erythrocytes was evaporated to dryness under nitrogen gas. Boron trifluoride-methanol solution $14 \%$ $(2 \mathrm{~mL})$ and methanol $(1 \mathrm{~mL})$ were added to the dried erythrocytes and heated in a Bain Marie for $10 \mathrm{~min}$ at $60^{\circ} \mathrm{C}$. Next, $2 \mathrm{~mL}$ of $\mathrm{n}$ hexane was added to each tube and shaken for $2 \mathrm{~min}$. After settling, the n-hexane layer containing the methylated fatty acids was transferred into another tube and the solvent was removed by evaporation. The residue was redissolved in $50 \mu$ of $n$-hexane, mixed thoroughly, and then $1 \mu \mathrm{l}$ of this solution was injected into the gas chromatograph. A gas chromatograph (YL6500; Young Lin, Korea) equipped with a $60 \mathrm{~m} \times 0.25 \mathrm{~mm}$ (film thickness $=0.2 \mu \mathrm{m}$ ) capillary column (TR-CN100; Teknokroma) and a flame ionization detector were used to measure the erythrocyte membrane fatty acid profiles. The YL Autochro3000 chromatograph data system v.2.0.15 was used for quantification and the identification of peaks. Fatty acid was expressed as the percentage of the total fatty acid curve area in the chromatogram. The DNL index (ratio of palmitate to linoleate) was also calculated.

\section{Statistical Analysis}

The data are presented as the mean \pm standard deviation (SD) and the median with interquartile range for parametric and nonparametric variables, respectively. The Shapiro-Wilks test was used to check the normality of the continuous variables and arithmetic transformations were performed if necessary. Continuous variables were compared between sexes by $t$ test.

Univariate linear regression was used to evaluate the association between serum NAMPT as an independent variable and CAP, erythrocyte membrane palmitic acid, and DNL index as dependent variables. Univariate linear regression was also used to evaluate the association between erythrocyte membrane palmitic acid and the DNL index as independent variables and CAP and body fat mass as dependent variables. These relationships were adjusted for factors that could affect DNL [23], including carbohydrate and protein intake, BMI (marker of positive energy balance), HOMA index, age and physical activity level using multiple linear regression (backward method). Variables were considered significant at $p<$ 0.2 for univariate linear regression and at $p<0.05$ for multiple linear regression. SPSS software version 23 was used to analyze the data.

\section{Results}

The baseline characteristics of the participants are shown in Table 1. The serum NAMPT, erythrocyte membrane DNL index and palmitic acid level, dietary protein, carbohydrate intake, fasting blood sugar, fasting serum insulin, physical activity level, and HOMA index were not significantly different between men and women.

In the overall analysis, both univariate and multiple linear regression analyses showed that serum NAMPT 
Table 1. Baseline characteristics of the participants

\begin{tabular}{|c|c|c|c|c|}
\hline Variables & $\operatorname{Men}(n=32)$ & Women $(n=30)$ & Total $(N=62)$ & $p$ value \\
\hline Age, years & $35.5(29-52)$ & $51(42.75-55)$ & $46.50(32.75-53.00)$ & 0.009 \\
\hline BMI & $29.43 \pm 4.89$ & $32.32 \pm 4.56$ & $30.83 \pm 4.91$ & 0.02 \\
\hline $\mathrm{W} / \mathrm{H}$ ratio & $0.89(0.87-0.94)$ & $0.86(0.84-0.88)$ & $0.88(0.85-0.91)$ & 0.024 \\
\hline $\mathrm{FBS}, \mathrm{mg} / \mathrm{dL}$ & $99(94-109)$ & $106.5(98.75-123)$ & $101(95-114.75)$ & 0.13 \\
\hline Serum insulin, $\mu \mathrm{IU} / \mathrm{mL}$ & $10.45(6.22-17.52)$ & $10.95(8.35-14.15)$ & $10.65(7.9-15.62)$ & 0.77 \\
\hline HOMA index & $2.67(1.5-4.58)$ & $3.06(2.05-3.79)$ & $2.77(1.90-3.96)$ & 0.53 \\
\hline Dietary $\mathrm{CHO}, \%$ total kcal & $53.16 \pm 5.02$ & $50.31 \pm 6.05$ & $51.95 \pm 5.61$ & 0.065 \\
\hline Dietary PRO, \% total kcal & $13.51 \pm 2.04$ & $12.45 \pm 2.16$ & $13.06 \pm 2.14$ & 0.07 \\
\hline CAP & $300(261.25-329)$ & $326(300-340)$ & $322.50(288.75-330.00)$ & 0.002 \\
\hline $\mathrm{LSM}, \mathrm{kPa}$ & $6.10(5-8.37)$ & $7(5.65-8.50)$ & $7(5-8.5)$ & 0.13 \\
\hline Body fat mass, $\%$ & $26.60 \pm 5.70)$ & $37.71 \pm 3.80$ & $31.97 \pm 7.39$ & 0.000 \\
\hline Serum NAMPT, ng/mL & $2.44 \pm 1.03$ & $2.45 \pm 1.13$ & $2.47(1.52-3.29)$ & 0.98 \\
\hline Erythrocyte membrane palmitic acid, \% & $20.55(15.34-24.04)$ & $22.64(21.15-25.95)$ & $22.27(18.59-25.012)$ & 0.07 \\
\hline DNL index & $3.11(2.60-3.71)$ & $3.05(2.40-3.59)$ & $3.08(2.53-3.64)$ & 0.90 \\
\hline Physical activity, MET-min/week & $924(350.62-6,432)$ & $693(247-1,854)$ & $793(294.75-3,183)$ & 0.067 \\
\hline
\end{tabular}

Data are presented as mean \pm SD for variables with normal distribution and median (interquartile range) for other variables. BMI, body mass index; CAP, controlled attenuation parameter; $\mathrm{CHO}$, carbohydrate; DNL, de novo lipogenesis; FBS, fasting blood sugar; HOMA, homeostasis model assessment; LSM, liver stiffness measurement; MET, metabolic equivalent of task; NAMPT, nicotinamide phosphoribosyltransferase; $\mathrm{PRO}$, protein; $\mathrm{W} / \mathrm{H}$ ratio, waist to hip ratio.

Table 2. Univariate linear regression of serum NAMPT with fatty acids of the erythrocyte membrane

\begin{tabular}{|c|c|c|c|c|c|c|c|c|c|c|c|c|}
\hline & \multicolumn{4}{|c|}{$\operatorname{Men}(n=32)$} & \multicolumn{4}{|c|}{ Women $(n=30)$} & \multicolumn{4}{|c|}{ Total $(N=62)$} \\
\hline & \multicolumn{2}{|c|}{ DNL index } & \multicolumn{2}{|c|}{ palmitic acid } & \multicolumn{2}{|c|}{ DNL index } & \multicolumn{2}{|c|}{ palmitic acid } & \multicolumn{2}{|c|}{ DNL index } & \multicolumn{2}{|c|}{ palmitic acid } \\
\hline & $\beta$ & $p$ & $\beta$ & $p$ & $\beta$ & $p$ & $\beta$ & $p$ & $\beta$ & $p$ & $\beta$ & $p$ \\
\hline Serum NAMPT & -0.17 & 0.38 & 0.17 & 0.36 & -0.44 & 0.03 & 0.054 & 0.79 & -0.33 & 0.02 & 0.11 & 0.41 \\
\hline Age & 0.13 & 0.50 & 0.24 & 0.20 & -0.03 & 0.89 & 0.008 & 0.97 & 0.04 & 0.76 & 0.20 & 0.13 \\
\hline BMI & -0.018 & 0.93 & -0.012 & 0.95 & -0.34 & 0.10 & -0.17 & 0.40 & -0.17 & 0.21 & 0.00 & 1 \\
\hline HOMA & 0.07 & 0.72 & 0.002 & 0.99 & 0.08 & 0.72 & -0.015 & 0.45 & 0.07 & 0.63 & -0.04 & 0.77 \\
\hline Dietary $\mathrm{CHO}$ & 0.21 & 0.30 & 0.10 & 0.59 & 0.20 & 0.42 & 0.20 & 0.39 & 0.22 & 0.15 & 0.02 & 0.90 \\
\hline Dietary PRO & 0.08 & 0.69 & -0.28 & 0.14 & 0.35 & 0.14 & 0.15 & 0.53 & 0.22 & 0.15 & -0.23 & 0.11 \\
\hline Physical activity & 0.26 & 0.19 & 0.04 & 0.82 & -0.04 & 0.86 & -0.07 & 0.75 & 0.13 & 0.36 & -0.03 & 0.81 \\
\hline
\end{tabular}

$\beta, \beta$ coefficient; BMI, body mass index; $\mathrm{CHO}$, carbohydrate; DNL, de novo lipogenesis; HOMA, homeostasis model assessment; NAMPT, nicotinamide phosphoribosyltransferase; PRO, protein.

had a significant negative association with DNL index $(\beta=-0.33, p=0.02$, and $\beta=-0.42, p=0.006$, respectively; Tables 2, 3). Serum NAMPT was not associated with erythrocyte membrane palmitic acid in either the univariate or multiple linear regression (Table 2).

In women, serum NAMPT had a significant negative association with DNL index for both univariate $(\beta=$ $-0.44, p=0.03$; Table 2$)$ and multiple $(\beta=-0.56, p=0.01$; Table 3 ) linear regression, but had no significant association with erythrocyte membrane palmitic acid level (Ta- ble 2). In men, there was no significant association between serum NAMPT and the erythrocyte membrane DNL index or palmitic acid level (Table 2).

Serum NAMPT was not significantly associated with hepatic fat content (CAP) in the overall analysis and in women (Table 4). However, in men it had a significant positive association with hepatic fat content (CAP) which was not significant in the univariate linear regression $(\beta=0.33, p=0.07$; Table 4$)$ but became significant in the multiple linear regression $(\beta=0.35, p=0.035$; Table 3$)$. 
Table 3. Multiple linear regression of serum NAMPT, CAP, body fat mass, and erythrocyte membrane fatty acids

\begin{tabular}{|c|c|c|c|c|c|c|c|c|c|c|}
\hline & \multirow{2}{*}{\multicolumn{2}{|c|}{$\begin{array}{l}\text { Men }(n=32) \\
\text { CAP }\end{array}$}} & \multicolumn{4}{|c|}{ Women $(n=30)$} & \multicolumn{4}{|c|}{ Total $(N=62)$} \\
\hline & & & \multicolumn{2}{|c|}{ body fat mass } & \multicolumn{2}{|c|}{ DNL index } & \multicolumn{2}{|c|}{ DNL index } & \multicolumn{2}{|l|}{ CAP } \\
\hline & $\beta$ & $p$ & $\beta$ & $p$ & $\beta$ & $p$ & $\beta$ & $p$ & $\beta$ & $p$ \\
\hline Serum NAMPT & 0.35 & 0.035 & - & - & -0.56 & 0.01 & -0.42 & 0.006 & - & - \\
\hline DNL index & - & - & -0.46 & 0.02 & - & - & - & - & - & - \\
\hline Palmitic acid & - & - & - & - & - & - & - & - & 0.29 & 0.03 \\
\hline
\end{tabular}

$\beta, \beta$ coefficient; CAP, controlled attenuation parameter; DNL, de novo lipogenesis; NAMPT, nicotinamide phosphoribosyltransferase.

Table 4. Univariate linear regression analysis of serum NAMPT and erythrocyte membrane fatty acids with CAP and body fat mass

\begin{tabular}{|c|c|c|c|c|c|c|c|c|c|c|c|c|}
\hline & \multicolumn{4}{|c|}{ Men $(n=32)$} & \multicolumn{4}{|c|}{ Women $(n=30)$} & \multicolumn{4}{|c|}{ Total $(N=62)$} \\
\hline & \multicolumn{2}{|c|}{ body fat mass } & \multicolumn{2}{|l|}{ CAP } & \multicolumn{2}{|c|}{ body fat mass } & \multicolumn{2}{|l|}{ CAP } & \multicolumn{2}{|c|}{ body fat mass } & \multicolumn{2}{|l|}{ CAP } \\
\hline & $\beta$ & $p$ & $\beta$ & $p$ & $\beta$ & $p$ & $\beta$ & $p$ & $\beta$ & $p$ & $\beta$ & $p$ \\
\hline Serum NAMPT & 0.20 & 0.27 & 0.33 & 0.07 & 0.075 & 0.69 & -0.01 & 0.61 & 0.10 & 0.45 & 0.10 & 0.401 \\
\hline DNL index & -0.02 & 0.91 & 0.09 & 0.66 & -0.46 & 0.02 & 0.26 & 0.21 & -0.11 & 0.45 & 0.17 & 0.23 \\
\hline Palmitic acid & -0.03 & 0.88 & 0.26 & 0.16 & 0.10 & 0.64 & 0.31 & 0.13 & 0.17 & 0.22 & 0.33 & 0.01 \\
\hline Age & -0.014 & 0.94 & 0.10 & 0.58 & -0.012 & 0.95 & 0.32 & 0.09 & 0.25 & 0.05 & 0.30 & 0.018 \\
\hline BMI & 0.80 & 0.00 & 0.30 & 0.09 & 0.60 & 0.00 & 0.20 & 0.28 & 0.67 & 0.00 & 0.34 & 0.007 \\
\hline HOMA & 0.48 & 0.005 & 0.04 & 0.04 & 0.12 & 0.54 & -0.06 & 0.76 & 0.24 & 0.06 & 0.19 & 0.126 \\
\hline Dietary $\mathrm{CHO}$ & -0.47 & 0.008 & -0.08 & 0.69 & -0.17 & 0.432 & 0.21 & 0.33 & -0.41 & 0.002 & -0.05 & 0.717 \\
\hline Dietary PRO & -0.10 & 0.61 & -0.1 & 0.59 & -0.11 & 0.60 & -0.22 & 0.30 & -0.25 & 0.07 & -0.23 & 0.095 \\
\hline Physical activity & -0.45 & 0.01 & -0.06 & 0.77 & -0.10 & 0.63 & 0.20 & 0.31 & -0.42 & 0.001 & -0.09 & 0.50 \\
\hline
\end{tabular}

$\beta, \beta$ coefficient; BMI, body mass index; CAP, controlled attenuation parameter; CHO, carbohydrate; DNL, de novo lipogenesis; HOMA, homeostasis model assessment; NAMPT, nicotinamide phosphoribosyltransferas; PRO, protein.

Serum NAMPT was not significantly associated with body fat mass in the overall analysis or for either sex separately (Table 4).

The DNL index had a significant negative association with body fat mass only in women in univariate and multiple linear regression $(\beta=-0.46, p=0.02$; Table 3,4$)$. It had no significant association with hepatic fat content (CAP) in men, women or in the overall analysis (Table 4).

Palmitic acid of the erythrocyte membrane had a significant positive association with hepatic fat content (CAP) only in the overall analysis for univariate linear regression $(\beta=0.33, p=0.01$; Table 4$)$ and multiple linear regression $(\beta=0.29, p=0.03$; Table 3$)$. It had no significant association with body fat mass in the overall analysis or for either sex (Table 4).

\section{Discussion}

In this study serum NAMPT had a significant negative association with the DNL index of the erythrocyte membrane. However, gender analysis revealed that the significant association was retained only in women and not in men. The DNL index also showed a significant negative association with body fat mass only in women.

These findings indicate that higher serum NAMPT is associated with lower hepatic DNL in women. Recent studies indicate that hepatic DNL and adipose tissue DNL are 2 pathways that support metabolic homeostasis and when one decreases the other increases to sustain blood glucose homeostasis $[12,24]$. In this study, it could be speculated that higher serum NAMPT in women might also be associated with a higher adipose tissue DNL. This speculation is strengthened by the significant

Med Princ Pract 2017;26:251-257 DOI: $10.1159 / 000455862$ 
negative association between DNL index (a marker of hepatic DNL) and body fat mass in women in this study. A higher fat mass in women and the positive effect of estrogen on NAMPT expression in 3T3-L1 cells [25] may result in a stronger association between serum NAMPT (an adipokine) and adipose tissue DNL in women.

The only significant association in men was between serum NAMPT and hepatic fat content. This indicated that the positive association between the serum NAMPT level and hepatic fat content in men might be more related to the inflammatory role of NAMPT $[26,27]$ than its role in hepatic DNL. The lack of a significant association between serum NAMPT and palmitic acid in the erythrocyte membrane in the overall analysis and in either sex can probably be explained as follows. Palmitic acid which is the main product of DNL and linoleic acid is an essential fatty acid for which the only source is dietary intake. A diet rich in linoleic acid is poor in saturated fatty acids, including palmitic acid, so when this ratio is increased it can be more related to the de novo synthesis of palmitic acid than to palmitic acid in the diet. Therefore, this ratio is a stronger marker for DNL than palmitic acid alone.

The results show that higher serum NAMPT in women indicates a lower hepatic DNL and possibly higher adipose tissue DNL. In men, an increase in serum NAMPT could be more strongly related to an increased hepatic fat content and the resulting inflammation.

Previous studies have shown that hepatic DNL and adipose tissue DNL have opposing effects on the prognosis of NAFLD. Hepatic DNL is positively correlated with insulin resistance and a fatty liver, but adipose tissue DNL is negatively correlated with these 2 factors $[12,28]$. Therefore, the serum NAMPT level might have a different prognosis for each sex and the contrary findings for the association between serum NAMPT and pathologic findings of NAFLD [29] could be partly due to the sex distribution in various studies. It appears that NAMPT function could be affected by sex, which is in accordance with a previous study that showed NAMPT is regulated by sex hormones in adipocytes [30]. In this study, the associations were adjusted for variables that could stimulate DNL [23], including carbohydrate and protein intake, BMI (marker of positive energy balance), HOMA index and age, so that the independence of associations could be confirmed.

The limitations of this study included its cross-sectional design and small population sample size. The intracellular pool of NAMPT may better reflect its effects in tissues than the extracellular pool so direct measurement of NAMPT and DNL activity in hepatic and adipose tissue may further clarify this association.

\section{Conclusions}

In this study, higher serum NAMPT was detected in women than men and it was significantly associated with a lower hepatic DNL index. Moreover, a lower hepatic DNL index was significantly associated with a higher body fat mass. Higher serum NAMPT was significantly associated with a higher hepatic fat content in men, and there were no significant associations between serum NAMPT, hepatic DNL index or body fat mass. Hence, the higher serum NAMPT in women could indicate lower hepatic DNL and possibly higher adipose tissue DNL, while in men NAMPT was related to a higher hepatic fat content and its inflammatory response. Therefore, the serum NAMPT level could have a different prognosis for each sex because of the opposing effects of hepatic and adipose tissue DNL on the prognosis of NAFLD.

\section{Acknowledgment}

This study was supported by Iran University of Medical Sciences, Tehran, Iran.

\section{Disclosure Statement}

The authors have no conflicts of interest to disclose.

\begin{tabular}{|c|c|c|}
\hline References & 3 & $\begin{array}{l}\text { Younossi ZM, Stepanova M, Afendy M, et al: } \\
\text { Changes in the prevalence of the most com- } \\
\text { mon causes of chronic liver diseases in the } \\
\text { United States from } 1988 \text { to } 2008 \text {. Clin Gastro- } \\
\text { enterol Hepatol 2011;9:524-530. } \\
\text { Fan JG: Epidemiology of alcoholic and nonal- } \\
\text { coholic fatty liver disease in China. J Gastro- } \\
\text { enterol Hepatol 2013;28:11-17. } \\
\text { Liu Q, Bengmark S, Qu S: The role of hepatic } \\
\text { fat accumulation in pathogenesis of non-alco- } \\
\text { holic fatty liver disease (NAFLD). Lipids } \\
\text { Health Dis 2010;9:1. } \\
\text { Turati F, Talamini R, Pelucchi C, et al: Meta- } \\
\text { bolic syndrome and hepatocellular carcinoma } \\
\text { risk. Br J Cancer 2013;108:222-228. } \\
\text { Lee JJ, Lambert JE, Hovhannisyan Y, et al: Pal- } \\
\text { mitoleic acid is elevated in fatty liver disease } \\
\text { and reflects hepatic lipogenesis. Am J Clin } \\
\text { Nutr 2015;101:34-43. }\end{array}$ \\
\hline
\end{tabular}

Amirkalali et al. 
6 Berlanga A, Guiu-Jurado E, Porras JA, et al: Molecular pathways in non-alcoholic fatty liver disease. Clin Exp Gastroenterol 2014;7: 221-239.

7 Hallows WC, Lee S, Denu JM: Sirtuins deacetylate and activate mammalian acetyl-CoA synthetases. Proc Natl Acad Sci USA 2006; 103:10230-10235.

8 Samal B, Sun Y, Stearns G, et al: Cloning and characterization of the cDNA encoding a novel human pre-B-cell colony-enhancing factor. Mol Cell Biol 1994;14:1431-1437.

9 Yang H, Lavu S, Sinclair DA: Nampt/PBEF/ Visfatin: a regulator of mammalian health and longevity? Exp Gerontol 2006;41:718726.

10 Yang C, Deng S, Hsu C, et al: Visfatin regulates genes related to lipid metabolism in porcine adipocytes. J Anim Sci 2010;88:32333241.

11 Bowlby SC, Thomas MJ, D’Agostino RB Jr, et al: Nicotinamide phosphoribosyl transferase (Nampt) is required for de novo lipogenesis in tumor cells. PLoS One 2012;7:e40195.

12 Solinas G, Borén J, Dulloo AG: De novo lipogenesis in metabolic homeostasis: more friend than foe? Mol Metab 2015;4:367-377.

13 Elizondo A, Araya J, Rodrigo R, et al: Polyunsaturated fatty acid pattern in liver and erythrocyte phospholipids from obese patients. Obesity 2007;15:24-31.

14 Chong MF, Hodson L, Bickerton AS, et al: Parallel activation of de novo lipogenesis and stearoyl-CoA desaturase activity after $3 \mathrm{~d}$ of high-carbohydrate feeding. Am J Clin Nutr 2008;87:817-823.
15 Dalamaga M: Nicotinamide phosphoribosyltransferase/visfatin: a missing link between overweight/obesity and postmenopausal breast cancer? Potential preventive and therapeutic perspectives and challenges. Med Hypotheses 2012;79:617-621.

16 Sasso M, Tengher-Barna I, Ziol M, et al: Novel controlled attenuation parameter for noninvasive assessment of steatosis using Fibroscan ${ }^{\circledR}$ : validation in chronic hepatitis C. J Viral Hepat 2012;19:244-253.

17 Sandrin L, Fourquet B, Hasquenoph J-M, et al: Transient elastography: a new noninvasive method for assessment of hepatic fibrosis. Ultrasound Med Biol 2003;29:1705-1713.

18 Sanyal AJ: AGA technical review on nonalcoholic fatty liver disease. Gastroenterology 2002; 123:1705-1725.

19 Hudgins LC, Hellerstein M, Seidman C, et al: Human fatty acid synthesis is stimulated by a eucaloric low fat, high carbohydrate diet. J Clin Invest 1996;97:2081.

20 Esmaillzadeh A, Mirmiran P, Azizi F: Wholegrain intake and the prevalence of hypertriglyceridemic waist phenotype in Tehranian adults. Am J Clin Nutr 2005;81:55-63.

21 Booth ML, Ainsworth BE, Pratt M, et al: International physical activity questionnaire: 12 -country reliability and validity. Med Sci Sports Exerc 2003;195:3508-1381.

22 McDowell MA: Anthropometric reference data for children and adults: US population, 1999-2002. US Department of Health and Human Services, Centers for Disease Control and Prevention, National Center for Health Statistics, 2005.
23 Hellerstein MK, Schwarz J-M, Neese RA: Regulation of hepatic de novo lipogenesis in humans. Annu Rev Nutr 1996;16:523-557.

24 Kuriyama H, Liang G, Engelking LJ, et al: Compensatory increase in fatty acid synthesis in adipose tissue of mice with conditional deficiency of SCAP in liver. Cell Metab 2005;1: 41-51.

25 Zhou J, Seidel ER: Estrogens induce visfatin expression in 3T3-L1 cells. Peptides 2010;31: 271-274.

26 Moschen AR, Kaser A, Enrich B, et al: Visfatin, an adipocytokine with proinflammatory and immunomodulating properties. J Immunol 2007;178:1748-1758.

27 Jia SH, Li Y, Parodo J, et al: Pre-B cell colonyenhancing factor inhibits neutrophil apoptosis in experimental inflammation and clinical sepsis. J Clin Invest 2004;113:1318-1327.

28 Minehira K, Vega N, Vidal H, et al: Effect of carbohydrate overfeeding on whole body macronutrient metabolism and expression of lipogenic enzymes in adipose tissue of lean and overweight humans. Int $J$ Obes Relat Metab Disord 2004;28:1291-1298.

29 Bekaert M, Verhelst X, Geerts A, et al: Association of recently described adipokines with liver histology in biopsy-proven non-alcoholic fatty liver disease: a systematic review. Obes Rev 2016;17:68-80.

30 MacLaren R, Cui W, Cianflone K: Visfatin expression is hormonally regulated by metabolic and sex hormones in 3T3-L1 pre-adipocytes and adipocytes. Diabetes Obes Metab 2007;9:490-497. 